\title{
I germanismi nello spazio linguistico della penisola italica: superstrato prodotto dalla migrazione; ambito di analisi diacronica, diatopica, e stilistica; strumento per la didattica.
}

\author{
Giovanni Fort (Umeå Universitet, Svezia)
}

\begin{abstract}
Germanic peoples appear strongly on the stage of history during late antiquity. With the advent of so-called "Barbarian Invasions" (or "folk migrations", if the perspective is that of the invaders), raids by Germanic tribes gradually turn into migrations of ethnic groups settling in the areas they strike. With the fall of the Empire and the creation of Barbarian Kingdoms, this phenomenon leads to lasting effects on local languacultures. In the Italian peninsula, Goths, Langobards, and Franks, impacted the evolution of vulgar Latin, leaving visible traces in the Italian language.

The Germanic element of Italian vocabulary is represented by a multitude of toponyms and anthroponyms; it characterises specific lexical areas, and is observable in basic vocabulary and derivational morphology. These elements (systematically collected within the LEI project) are an extremely interesting object of study, on several levels.

In a diachronic perspective: analysing their presence at different stages, and as an instrument for dating.

In a diatopic perspective: as a criterion of dialectological analysis, also frequently linked to geosynonyms and so-called "parole bandiera". (Besides also being a differentiating criterion between romance languages).

In a sociolinguistic and stylistic perspective: considering the value of a Latin or a Germanic equivalent, in context.

It is moreover ultimately relevant to consider an approach involving Germanic elements in Italian as an effective pedagogical tool. They can prove extremely useful, not only in educating about the history of the languaculture of the Italian peninsula, but also in teaching basic language-competence, and in the expansion of vocabulary, exploiting intercomprehension in learners with a Germanic mother tongue in general, and a Scandinavian one in particular (and vice versa).
\end{abstract}

Keywords: Italian, ancient germanic, loanwords, intercomprehension, philology

\section{Introduzione}

Sono oggi di pressante attualità riflessioni sui fenomeni interculturali che riguardano spazio e migrazione. In un mondo sempre più globalizzato in cui stanno avendo luogo grandi spostamenti di persone, che assieme ai media favoriscono la diffusione dei prodotti linguaculturali di diversi popoli ben al di là dei confini nazionali, si rinnova nelle nostre 
discipline l'interesse per l'analisi delle modalità e degli impatti di questo tipo di scambi. Laddove ci è dato solo di navigare a vista nel presente, cercando di leggere gli auspici che indicano le direzioni delle evoluzioni future, possiamo invece con profitto rivolgere lo sguardo a ciò che già è stato, alla ricerca di fenomeni isomorfi capaci non solo di aiutarci nell'analisi di ciò che è e di ciò che verrà, ma anche di procurarci una visione più nitida e sfaccettata di un passato che potremmo altrimenti invece tendere a percepire come monolitico.

Un tema che ben si presta a questo tipo di operazione è quello dei germanismi nello spazio linguistico della penisola italica. Lo studio di questo superstrato prodotto dalla migrazione è per propria natura interdisciplinare, combinando le aree di interesse della filologia romanza e della filologia germanica, che qui possono entrare in fruttuosa sinergia. Questa porzione del lessico italiano, seconda per entità solo al fondo latino, ben si presta inoltre ad essere strumento e criterio di analisi linguistica sul piano diacronico, diatopico e dialettologico, sociolinguistico e stilistico. È infine rilevante la possibilità di sfruttare un approccio ai germanismi della lingua italiana come strumento privilegiato nella didattica, non solo della storia della lingua e della cultura, ma anche della competenza lessicale di base, nell'ambito dell'insegnamento a studenti di madrelingua germanica in generale, e scandinava in particolare: questo tipo di operazione è attuabile facendo leva sui fenomeni di intercomprensione attivabili nei discenti, a posteriori di una introduzione ai fenomeni di trasformazione diacronica, ed è riproponibile nella direzione opposta anche presso discenti di madrelingua italiana che si approcciano a una lingua germanica o scandinava.

È intenzione dunque di questo scritto mettere a fuoco e descrivere il fenomeno, indicarne le possibili applicazioni come strumento in diversi ambiti, e delineare lo stato attuale degli studi e le prospettive per ulteriori lavori futuri, alla luce delle potenzialità che l'oggetto di studio offre.

\section{Le origini storiche dei germanismi nella lingua italiana}

Per inquadrare i germanismi nello spazio linguistico della penisola italica è utile delineare i fenomeni storici di contatto con i popoli germanici a cui il fenomeno linguistico di superstrato è dovuto.

Le fonti storiche ci indicano contatti anche in epoca antica tra i Latini e i Germani. La presenza significativa di questi ultimi sul territorio italiano, capace di influenze linguistiche, è da individuarsi però principalmente nell'arco di quattro secoli, tra il V e l'VIII, a cavallo tra la tarda antichità e il medioevo.

I Germani, coinvolti già da tempo nel fenomeno del periodo delle grandi migrazioni, cominciarono a penetrare in Italia ben prima della caduta dell'impero romano: arruolati come mercenari occuparono gradualmente sempre più numerosi i ranghi dell'esercito, arrivando progressivamente anche ad alte cariche e alla periferia del potere politico. Con il sistema di attribuzione di terre pubbliche in cambio della disponibilità alla chiamata alle armi, già nel IV secolo si riscontrano nella penisola forme di insediamento paramilitare di gruppi di diverse etnie germaniche.

Nel V secolo il ruolo di alleati e mercenari diventa progressivamente intercambiabile con quello di invasori più o meno stabili a seconda delle vicissitudini politiche: emblematico di questo fenomeno è l'esempio dei Visigoti nel V secolo, dapprima federati dell'impero, e poi invasori della penisola e autori, sotto la guida di Alarico, del sacco di Roma del 410. 
L'evento stesso che segna la caduta dell'impero romano d'occidente è opera di una sollevazione di federati di diverse etnie germaniche, che guidati da Odoacre depongono nel 476 l'imperatore Romolo Augustolo. Odoacre invia le insegne imperiali a Costantinopoli ed assume il potere come re delle genti germaniche stanziate in Italia.

Questo momento demarca l'inizio del periodo dei cosiddetti regni romano-barbarici, che a partire dal V secolo rappresentano in Italia (ma anche nella Gallia, nella Britannia, nella penisola iberica e nel Nordafrica) l'appagamento delle esigenze territoriali dei Germani che si afferma sulle rovine dell'impero.

Nel 476 le invasioni barbariche assumono la forma di migrazioni stanziali corrispondenti anche a degli assetti politici nella penisola italica, raggiungendo il proprio culmine.

Le dominazioni e le etnie germaniche che soprattutto segnarono la storia e la lingua italiana nel periodo dal V all'VIII secolo sono due: quella gotica, con l'invasione di Teodorico nel 489 e il regno ostrogotico (493-553), e quella longobarda (569-774). Il regno longobardo terminerà ad opera di un terzo popolo germanico, a questo punto però già ampiamente romanizzato: i Franchi di Carlo Magno.

Non è questa la sede per una trattazione di carattere storicoculturale dei regni romanobarbarici che interessarono la penisola italica, e per essa si rimanda ad opere di taglio specialistico in questo senso ${ }^{1}$. È però utile sottolineare brevemente alcune caratteristiche che saranno funzionali alle riflessioni del presente lavoro.

Da un punto di vista geografico, l'occupazione dei Goti fu molto estesa: interessò le regioni dell'Italia del nord, le regioni lungo l'Appennino centrale e meridionale e quasi tutta la penisola. Da un punto di vista demografico fu invece relativamente ridotta: si stima in termini di massimo 200.000 individui a fronte di una popolazione italica superiore ai 4 milioni (attorno quindi al 5\% rispetto al 20\% dei Franchi stanziati nella Gallia settentrionale) (Bonfante 1977, 12). I regni gotici furono improntati a una separazione di ordine politico e amministrativo tra le due etnie, in una sorta di dualismo orientato alla tolleranza. Sulla base di questi dati e delle fonti che ci sono rimaste, si conclude che il fenomeno di superstrato non sfociò probabilmente in fenomeni diffusi di diglossia o di bilinguismo nelle popolazioni italiche $^{2}$.

La migrazione longobarda si sviluppò in parallelo agli insediamenti militari (dato che $\mathrm{i}$ corpi dell'esercito le farae- di cui ancora rimane traccia nei toponimi italianicorrispondevano ai nuclei tribali) che presto si trasformarono in colonizzazioni stabili. Interessò soprattutto tre zone: Italia settentrionale (Pavia capitale), Toscana, e i territori centromeridionali di Fermo, Spoleto e Benevento. Anche da un punto di vista demografico la migrazione longobarda fu probabilmente più ridotta di quella gotica (stimata al massimo attorno alle 150.000 unità anche se a fronte di una popolazione italica che si stima attorno a 1 milione, molto più ridotta quindi di quella che trovarono i Goti al tempo della loro discesa) (Bonfante 1977, 24). Nonostante una estensione geografica e demografica più ridotta,

\footnotetext{
${ }^{1}$ Si veda ad esempio Morlicchio (2011) e Morlicchio (2001).

${ }^{2}$ Cfr. Morlicchio (2005) e Albano Leoni (1983).
} 
l'invasione longobarda riuscirà ad avere un influsso più decisivo di quello dei predecessori Goti ${ }^{3}$.

Una notevole invadenza dei termini longobardi nei settori della vita pubblica testimonia una situazione di quantomeno parziale diglossia se non bilinguismo. Questa influenza è testimoniata anche nella denominazione dell'Italia stessa. Italia è glossata Longobardia nelle glosse di Reichenau dell'VIII secolo ${ }^{4}$, e Carlo Magno stesso nel proprio testamento indica di lasciare a Pipino Italiam, quae et Langobardia dicitur. Questo uso sopravvivrà a lungo dopo la fine del regno longobardo, come testimonia il celebre esempio della Lombard Street di Londra, così chiamata per via della concessione che nel XIII secolo Edoardo I fece ai prestatori a pegno originari dell'Italia settentrionale, un'area che si estendeva ben al di là della regione odierna, la Lombardia, che ancora oggi dei dominatori germanici porta il nome.

La dominazione franca si sostituì a quella longobarda con la vittoria di Carlo Magno nel 774, e il regno cambiò nome da Regnum Langobardorum a Regnum Italiae. L'ordinamento esistente venne però mantenuto e gli editti dei re longobardi restarono in vigore affiancati ai successivi capitolari carolingi. La nobiltà franca si affiancò a quella longobarda sostituendola nei ruoli di potere politico, e solo allora progressivamente i Longobardi cominciarono ad assimilarsi alla popolazione italica che era stata loro soggetta, perdendo individualità culturale e linguistica. I Franchi giunsero in Italia, come si è detto, già ampiamente romanizzati per lingua e cultura ${ }^{5}$. La loro dominazione, a differenza delle invasioni germaniche precedenti, non rappresentò un fenomeno demograficamente significativo e costituì solo la penetrazione di una nuova classe dirigente in una società già stabile e organizzata.

\section{Le fonti dirette}

Laddove non mancano fonti latine dell'epoca riguardo a Goti e Longobardi (il $D e$ origine actibusque Getarum di Giordanes, e la Historia Langobardorum di Paolo Diacono) scarse sono invece le attestazioni delle lingue di questi popoli.

Testimonianze della lingua degli Ostrogoti ci sono state tramandate per lo più in glosse e antroponimi all'interno di testi e documenti latini, pubblici e privati. L'unica menzione diretta della penetrazione del gotico nella quotidianità dell'Italia tardolatina si ha nella Anthologia Latina, che alla metà del V secolo riporta "Inter 'eils'goticum 'scapia matzia ia drincan' non audet quisquam dignos educere uersus"'(epigramma 285, De conuiuiis barbaris) ${ }^{6}:$ l'autore lamenta che tra le grida gotiche che chiedono da mangiare e da bere nessuno più osa produrre versi decenti. Richiamo l'attenzione del lettore, che vista la destinazione di questo scritto avrà probabilmente familiarità con le lingue scandinave, sulla

\footnotetext{
${ }^{3}$ Come sottolinea Pfister $(1998,114)$, esistono studi esaustivi sul lessico del superstrato longobardo nell'area della Italoromania. Si veda ad esempio Mastrelli (1974), o Albano Leoni (1983) che a partire dai suoi studi propone anche nel 643 un convenzionale terminus ante quem per la fine del periodo linguistico longobardo.

${ }^{4}$ Digitalizzate e consultabili all'indirizzo https://digital.blb-karlsruhe.de/blbhs/content/titleinfo/192577. Data ultimo accesso 10-10-2017

${ }^{5}$ Il che pone la ulteriore necessità di operare distinzione tra i vocaboli che risalgono direttamente al superstrato germanico e quelli che invece sono esempi di prestiti interromanzi etimologicamente risalenti ad una origine francone (Pfister 1998, 1120-1121).

${ }^{6}$ Digitalizzato e consultabile su https://archive.org/details/pt1a2anthologial01buecuoft Data ultimo accesso 1010-2017
} 
possibilità di riconoscere con limitata difficoltà in quel "skapia matzia ia drincan" il corrispettivo svedese moderno skaffa mat och dricka, un tipo di riconoscimento su cui presto torneremo trattando della possibile applicazione dei germanismi come strumento didattico della lingua italiana per i parlanti di madrelingua scandinava.

Secondo gli studiosi, anche con stime divergenti, sarebbero relativamente pochi i germanismi di origine indubbiamente gotica, e apparterrebbero osservabilmente tutti al linguaggio quotidiano, mentre mancano invece tra $\mathrm{i}$ termini in ambito giuridico ed amministrativo, aree che rimasero forse prerogativa del latino, e in cui abbondarono poi invece i longobardismi (magari sostituendosi anche a goticismi precedenti o adattandoli linguisticamente).

Anche nel caso del longobardo le attestazioni dirette sono quasi esclusivamente di tipo lessicale. Si tratta ancora una volta di glosse in testi latini (documenti pubblici e privati, opere storiche e codici di leggi), glosse prevalentemente di ambito giuridico, patrimoniale, o riguardanti ruoli politici e sociali. Malgrado la scarsità di attestazioni dirette, gran parte dei germanismi penetrati nel volgare italiano vengono solitamente ricondotti al longobardo, secondo criteri storici e linguistici che andremo a breve ad esaminare.

\section{I germanismi nella Lingua Italiana}

Data la detta scarsità di attestazioni dirette, la testimonianza più forte che hanno lasciato queste popolazioni germaniche sta invece proprio negli elementi lessicali e morfologici penetrati nella lingua italiana e nei suoi dialetti, che sono l'oggetto di questo scritto.

Sulla base dei dati storici sopra delineati, gli studiosi riconoscono tradizionalmente (Molinari 1987, 187) tre strati principali di germanismi nella lingua italiana: gotico, longobardo e francone. Distinguere però tra i germanismi ed accertarne l'esatta origine è in molti casi cosa assai difficile, e come sottolinea Bonfante costituisce "uno dei problemi più ardui della linguistica romanza" (Bonfante 1977, 26). Anche per questo motivo si è soliti aggiungere alle precedenti tre categorie una quarta, quella di generali "superstrati germanici" per le voci penetrate nel latino volgare senza che si possa distinguere chiaramente da quali popoli germanici provengano (Pfister 1998, 1108) ${ }^{7}$.

Meno arduo però, e iscrivibile nella portata del presente lavoro, è individuare alcuni criteri di generale validità, che ci saranno di aiuto nel descrivere i germanismi penetrati nella lingua italiana e che entreranno anche in sinergia con il loro uso strumentale nella didattica che qui si vuole proporre.

Per quanto riguarda un riconoscimento a prima vista dei germanismi in italiano, è possibile indicare tre semplici criteri grafico-fonetici di generale anche se non assoluta efficacia.

Con rarissime eccezioni sono tutte germaniche le parole e gli elementi onomastici e toponomastici che cominciano con gu+vocale. Ne indicherò a seguire alcuni esempi, scelti sulla base di un criterio di facile identificazione del corrispettivo in una lingua germanica moderna: guadare (sv. vada), gualdo (ted. Wald), guancia (ted. Wange), guanto (sve. vante), guastare (ing. waste), guerra (ing. war), guisa (sve. vis), Gugliemo (sv. Vilhelm).

\footnotetext{
${ }^{7}$ Pfister $(1998,1108)$, discutendo della intera area romanza, ricorre a questa categoria soprattutto per le voci penetrate prima del 630, generalmente di origine gotica, ma non individuabile in gotica occidentale o orientale.
} 
Segnalano origine germanica le parole italiane che iniziano con sche- e schi-, dato che la pronuncia di sce- e sci- latini era già mutata per effetto della vocale palatale. Esempi, scelti col consueto criterio, sono: schermo (sv. skärm), scherzare (ted. scherzen), schiena (ing. shin, sv. skenben, con deriva semantica), schiniere (sv. skenben), schiuma (sv. skum), schizzare (ted. schiessen).

'Sono infine germaniche quasi tutte le parole italiane con z- sorda iniziale. Ad esempio zinna (sv. tinne), o zanna (ted. Zahn/sv. tand).

Per quanto riguarda la distinzione di appartenenza a uno dei tre principali strati di germanismi nella lingua italiana ${ }^{8}$, un criterio assai utile è la presenza o assenza della seconda mutazione consonantica altotedesca antica. Questo fenomeno, tipico del tedesco meridionale, riguardò il longobardo, ma non il gotico e il francone, che presentano quindi un consonantismo vicino a quello delle lingue scandinave o dell'inglese. Laddove non ci permette di distinguere un germanismo di origine franca da uno gotico, la presenza del fenomeno ci lascia individuare in molti casi gli etimi di origine longobarda.

La seconda mutazione consonantica, una evoluzione databile a partire dalla metà del V secolo, si manifesta con un cambiamento concatenato del modo, ma non del luogo, di articolazione delle occlusive sorde e sonore, e della spirante interdentale sorda. Nello specifico ci interessano qui i fenomeni riguardanti:

Le occlusive sorde, che diventano affricate o spiranti doppie: $p, t, k$ diventano $p f, t z / z$, $k h$, oppure $f f, z z[\mathrm{ss}], h h / c h$

Le occlusive sonore (e le rispettive spiranti sonore), che diventano occlusive sorde: $b, d, g$ diventano $p, t, k$

Ben illustrano le conseguenze del mutamento, ad esempio, coppie di parole moderne tedesche e svedesi come Zeit-tid, Pfeffer-peppar.

Applicando questo strumento ad una analisi dei germanismi del lessico italiano possiamo dedurre che saranno longobardi gli etimi germanici che presentano affricate sorde, o suoni derivati da affricate sorde.

È quindi gotico tetta (cfr. sv. tutte) e longobardo zizza, longobardo zaffo, e gotico tappo.

Similmente, secondo l'evoluzione delle occlusive sonore, è gotico balla ( $s v$. boll), mentre è longobardo palla; probabilmente gotico balcone ( $s v$. balk) e longobardo palco.

L'assenza della seconda mutazione consonantica non garantisce che non si tratti comunque di un germanismo longobardo. La presenza di questo fenomeno è invece chiaro indizio di parola italiana di origine longobarda, e la sua conoscenza può rivelarsi inoltre utile per il discente di madrelingua scandinava che si approccia allo studio della lingua italiana cercando agganci per l'intercomprensione.

\footnotetext{
${ }^{8} \mathrm{Si}$ è scelto di indicare nel testo i generali criteri di riconoscimento ad un primo approccio con elementi del lessico italiano. Criteri scientifici per l'individuazione di appartenenza dei prestiti germanici nelle lingue romanze sono: la presenza del prestito esclusivamente nell'Iberoromania, nel meridione della Francia ed in Italia per accertarne l'origine gotica (Pfister, 1998, 1109); le forme parallele nelle altre lingue germaniche, le fonti scritte longobarde redatte in latino medievale e la geografia linguistica moderna, per accertarne l'origine longobarda (Pfister, 1998, 1115).
} 
Applicando al contrario la seconda mutazione consonantica, sono agevolmente riconducibili al corrispettivo scandinavo alcuni tra i molti germanismi longobardi della lingua italiana. Ad esempio: aizzare (sv. hetsa), ciuffo (sv. tofs), milza (sv. mjälte), stronzo ( $s v$. strunt-), strozza (sv. (s)trut), spruzzare (sv. spruta), scaffale (sv. skåp), o zanna (sv. tand).

Le parole di origine gotica sono in numero più limitato: Bonfante ne individua $136 \mathrm{e}$ ne offre una lista (Bonfante 1977, 31-33) ${ }^{9}$. Ne elenco alcune, scelte sempre per la agevole avvicinabilità al corrispettivo nelle lingue germaniche e scandinave moderne:

Albergo (sv. härbärge), arredare (sv. in-reda) arringare (sv. här-ring), banda (sv. band), briglia (ing. bridle), elmo (ted. Helm/sv. hjälm), grappolo (sv. grepp), guardia (sv. vårdare) guitto (sv. vits), recare ( $s v$. räcka), rubare ( $s v$. röva), smaltire (sv. smälta), spiare ( $s v$. speja), stalla ( $s v$. stall), stecca/stocco ( $s v$. stake/stock), stanga (sv. stång), tasca ( $s v$. task(a)/ted. Tasche), tregua (sv. trygg).

\section{Aree del lessico interessate dai germanismi}

Delineati così alcuni aspetti che ci permettono di individuare e distinguere i germanismi penetrati nella lingua italiana, è rilevante passare a esaminare quali aree del lessico essi interessino.

Sulla base dei cenni storici prima esposti possiamo correttamente prevedere abbondanti gli antroponimi (con frequenza variabile sul piano diacronico e diatopico) e i toponimi.

Tra i molti antroponimi di origine germanica vi sono tanto quelli tuttora comuni e universalmente diffusi sul territorio (Alberto, Federico, Riccardo e Roberto, solo per menzionarne alcuni) quanto quelli con diffusione più specificamente regionale o di frequenza oggi più ridotta ${ }^{10}$ (ad esempio Berardo, Clotilde, Folco, Lapo, Gesualdo, Gisella, Gottardo, Rodolfo, Rolando, Uberto, Ugo(lino)).

L'asse diacronico è particolarmente interessante nell'evoluzione dell'onomastica: gli antichi antroponimi romani divennero sempre più infrequenti nel medioevo, sostituti da quelli germanici e quelli cristiani. Il picco massimo si ha attorno al X secolo, quando si calcola che circa il 75\% degli antroponimi nella penisola italica fossero germanici (Bonfante 1977, 58). Della permanenza di questa elevata frequenza si trova traccia evidente ad esempio negli antroponimi della Divina Commedia. Dante Alighieri stesso, una delle figure più famose associate alla penisola italica, testimonia col proprio cognome (assieme ad altri noti, come Garibaldi o Guinizzelli) l'entità dell'apporto del superstrato germanico alla nostra onomastica.

Coerentemente con gli eventi storici e la geografia dei regni romanobarbarici, i toponimi germanici hanno frequenza più ridotta dei preromani, romani e cristiani, e sono ristretti a determinate aree, ma sono comunque di entità significativa. Più scarsi, come ci si potrebbe aspettare, sono i toponimi gotici (esempi lampanti Goito, Gudi, Gudo e Sant'Agata dei Goti, dove sopravvive addirittura l'etnonimo, e tutta una serie di toponimi derivati da

\footnotetext{
${ }^{9}$ La lista proposta da Bonfante sulla base di quella del Brüch, si ridurrebbe sensibilmente applicando i criteri proposti da Pfister (1998, 1109), che ne farebbe rientrare massima parte nei "superstrati germanici" di origine non completamente specificabile, senza però negarne la generale appartenenza ad una fonte di influsso gotica. Cfr. qui nota 7 .

${ }^{10}$ Per alcuni notevoli esempi nell'ampio panorama di studi onomastici e antroponomastici in prospettiva più specificamente diacronica e diatopica si può fare ad esempio menzione di Morlicchio 1985 e 2012
} 
antroponimi, e.g. Gottolengo, Rodigo e Rovigo). Di frequenza assai maggiore, sono invece quelli longobardi: se ne contano centinaia derivanti da antroponimi, e molti altri basati su elementi lessicali di altro tipo: Ala (cfr. Halle), Breda, Sondrio (cfr.sv.sönder/sund), le molte Fara e Sala (cfr. sv.-sala), e Stongarda/Scottigarda (cfr. Stuttgart) nonché Garda, per menzionarne solo alcuni di significativi.

Sempre basandoci su quanto sappiamo dalla storia, ci aspetteremo di trovare una particolare influenza in aree specifiche del lessico relative al ruolo sociale e alle caratteristiche dei popoli germanici che in Italia si stanziarono ${ }^{11}$.

Ne troviamo conferma nei germanismi penetrati in ambito giuridico: alcuni usciti dall'uso linguistico con la scomparsa dell'uso nell'amministrazione della giustizia (si pensi al guidrigildo, ted. Widergeld, sv. vedergällning), altri ancora molto vivi anche nell'uso idiomatico (si pensi al fio - cfr. ing. fee- che ancora colloquialmente "paghiamo", o alla faida (sv. fejd).

Non sorprende anche il fatto che tanti siano i germanismi italiani legati alla "tecnologia del cavallo", più avanzata e cara presso i Germani che presso i Latini. Ne sono solo alcuni esempi parole come briglia (ing. bridle), maniscalco (sv. märr $+s k a l k$, sv. marskalk), sperone (sv. sporre), staffa (sv. stav), stalla (sv. stall).

Coerente col ruolo e le caratteristiche dei Germani in Italia è anche la elevata frequenza di germanismi nell'ambito collegato alla pratica della "germanicissima" guerra: alabarda, albergo, araldo, brando, elmo, gonfalone, guardia, guerra, rubare, schiera, sgherro, spalto, spiare, stormo, strale, e usbergo, sono solo alcuni fra molti esempi possibili di parole in questa area lessicale, alcune rimaste relegate al contesto storico specifico, altre sopravvissute nell'uso odierno.

Ancora più interessanti sono forse $\mathrm{i}$ casi di aree generiche del lessico in cui i germanismi penetrano, sostituendo l'alternativa latina preesistente, o affiancandosi ad essa con valenza leggermente diversa.

Troviamo germanismi nell'area lessicale che riguarda gli animali, particolarmente quelli legati alla caccia, riconducibili forse all'amore dei Germani per questa pratica. Ne sono esempi falco (sv. falk), becco (sv. bock), e stambecco (sv. stenbock).

I germanismi penetrano però, secondo logica meno ovvia, anche in aree come: il lessico delle parti del corpo umano (come: anca, guancia, milza, nocca, schiena, stinco e spanna), soppiantando sovente il termine latino, recuperato talora in latinismi colti, e.g. nel linguaggio della medicina; il lessico degli oggetti di uso comune, soprattutto di vestiario e arredamento, non sempre riconducibili a innovazioni germaniche (ne sono esempio banco, bara, brache, bucato, guanto, panca, scarpa e tasca); il lessico generico dei luoghi, come borgo, bosco, giardino, guado, gualdo, sala, stamberga.

La più estrema misura di questa penetrazione si riscontra però in alcune delle aree più fondamentali: il lessico delle qualità (come baldo, gramo, ricco, sghembo o snello) e addirittura il lessico dei colori, in cui i germanici bianco, biondo, blu, bruno, giallo, e grigio, vanno ad esempio a soppiantare i corrispettivi etimi latini.

\footnotetext{
${ }^{11}$ A conferma di quanto precedentemente sottolineato riguardo al più vasto ruolo giocato dal superstrato longobardo, si individuano ad esempio nelle fonti storiche circa 280 longobardismi solo nel lessico dei manufatti (Pfister, 1998, 114). Parte di essi è sopravvissuta nei dialetti e nell'uso della lingua moderna.
} 
Nemmeno la morfologia derivativa rimane indenne dall'influsso germanico, che ci ha dato in italiano i suffissi -esco, -ardo ed -ingo.

\section{I germanismi come elemento di differenziazione dalle altre lingue romanze}

La profondità della penetrazione del superstrato germanico nella lingua italiana è anche criterio di differenziazione tra le lingue romanze. La penisola iberica conobbe storicamente influssi germanici più ridotti di Francia e Italia, e questo è ben testimoniato proprio dal lessico di base.

$\mathrm{Ne}$ sono esempio diverse coppie di parole in cui lo spagnolo ha un etimo latino o di altra provenienza laddove italiano e francese (qui non indicato) ne hanno uno germanico: necesidad-bisogno (sv. be+sakna, ant.sv. be+sögna), azul-blu (sv. blå), rubio-biondo, amarillo-giallo (ing. yellow), costado-fianco ( $s v$. flank), sanar-guarire ( $s v . v a ̈ r j a), ~ m i r a r-$ guardare (sv. vårda), feo-laido (sv. lat).

Come ci si può aspettare alla luce di quanto già discusso sulla diversa proporzione demografica della migrazione germanica in Francia e in Italia, esistono anche molti esempi in cui è solo il francese ad avere un etimo germanico laddove italiano e spagnolo ne presentano di origine diversa.

Ci sono tuttavia casi, rari ma significativi, in cui solo l'italiano ha adottato il germanismo laddove spagnolo e francese hanno preso strade diverse: bugia-sp. mentira e fr. mensogne; guancia-sp. mejilla e fr. joue; milza-sp. bazo e fr. rate; schiena-sp. espalda e fr. dos.

\section{I germanismi come strumento e oggetto di analisi (diacronica, diatopica e sociolinguistica, stilistica)}

I germanismi si rivelano, come evidenziato, strumento e oggetto di analisi utile su un piano diatopico su tutta l'area della lingue romanze. Questo vale anche per la variazione diatopica interna alla penisola. Oltre alle differenze sul piano dei dialetti, i germanismi sono aspetto rilevante a livello di italiano standard, di uso medio, e regionale, approcciandosi ad esempio a fenomeni come quello dei geosinonimi, di rango anche nazionale, e delle cosiddette "parole bandiera".

La distribuzione dell'uso di due diverse parole per designare uno stesso oggetto, come ad esempio gruccia (sv. krycka)-appendiabiti, disegna una carta che divide la penisola in due Italie, una "germanica" e una "grecoromana". Proprio come la linea La Spezia-Rimini (o Massa-Senigallia) separa con un fascio di isoglosse le lingue romanze orientali da quelle occidentali e fa da spartiacque tra la macroregione dialettale italiana settentrionale e le altre ${ }^{12}$, così possiamo individuare una linea Roma-Pescara (Bonfante 1977, 43) ${ }^{13}$ ai due lati della quale si alternano presenza e assenza dei geosinonimi germanici, secondo coppie come: schiena/dorso, guarire/sanare, buttare (sv. putta)/gettare, giardino/orto, o guardare/mirare.

Spostandoci sugli altri assi di variazione è possibile utilizzare i germanismi come angolazione per riflessioni di tipo sociolinguistico, sia in prospettiva diacronica che

\footnotetext{
${ }^{12}$ Presentando inoltre secondo lo Pfister un fascio di isoglosse di impronta longobarda che ha avuto una certa funzione unificatrice tra la Pianura Padana e la Toscana (Pfister 1998, 1117).

${ }_{13}$ Altre linee di separazione fondamentali per la distribuzione areale dei lessemi di origine germanica sono quella Ancona- Colli Albani, e quella meridionale Salento-Calabria centrale (Pfister, 1998, 1117)
} 
sincronica: ci offrono una dimensione per analizzare la valenza del loro uso, in termini di variabile diastratica in generale, e di variabile diastratica collegata a quella diatopica soprattutto nel contesto postunitario.

$\mathrm{Si}$ interseca in maniera fruttuosa con una riflessione sui germanismi relativa ai vari assi di variazione anche un loro impiego come strumenti e oggetti di studio in ambiti tangenti a quelli della stilistica. In una fase della lingua in cui due alternative, una germanica e una latina, esistono contemporaneamente, che valenza e che tipo di connotazione ha optare per l'una o per l'altra? La scelta di addensare molti germanismi per caratterizzare un certo tipo di descrizioni, si riscontra ed è significativa e cosciente nel caso di autori particolarmente attenti alla lingua? Chi scrive ha condotto degli studi preliminari, di natura qualitativa e quantitativa, sulla Divina Commedia che sembrano indicare un terreno fertile in questo senso.

Anche solo da una analisi cursoria delle parole semanticamente collegate emerge una chiara tendenza ad utilizzare i germanismi con coloritura peggiorativa o intensificativa (come si osservava anche nell'uso del suffisso germanico -esco).

Si considerino ad esempio le connotazioni dei membri di coppie di parole come le seguenti: amante-drudo; capigliatura-zazzera; ceco-guercio; dente-zanna; bere-trincare; golastrozza; intelligenza-senno; morire-schiattare; naso-nappia; odio-astio, onestuomo-farabutto; pace-guerra; stomaco-magone.

Una analisi del lessico attraverso la lente dei germanismi apre prospettive promettenti, tanto per una riflessione sulla percezione socioculturale di questi popoli durante la permanenza in Italia, se impiegati come oggetto, quanto per riflessioni di natura stilistica, se impiegati come strumento.

Sempre in una ottica di sfruttamento dei germanismi come strumento, sul piano diacronico si aggiunge anche l'ovvio potenziale di impiego come terminus a quo nella datazione, e come criterio di individuazione della fase di sviluppo della lingua ${ }^{14}$.

\section{I germanismi come strumento nella didattica della lingua italiana: il caso scandinavo}

Tra gli impieghi potenziali che proiettano a mio avviso la tematica dei germanismi a pieno titolo come area meritoria di rinnovata attenzione nei nostri ambiti, vorrei sottoporre in questa sede in maniera particolare al lettore il caso della didattica universitaria della lingua italiana presso discenti di madrelingua germanica in generale, e scandinava in particolare.

Si sta ultimamente rivolgendo crescente attenzione ai fenomeni di intercomprensione anche nell'ambito della glottodidattica. Studi recenti hanno indagato e continuano a indagare $\mathrm{i}$ processi di intercomprensione di lingue che il partecipante non conosce, ma che appartengono alla stessa famiglia linguistica della lingua madre o di una lingua già appresa.

Sono stati attivati progetti europei di ampio respiro sull'intercomprensione, come EuroComRom e EuRom5 per le lingue romanze, o EuroComGerm per le lingue germaniche.

\footnotetext{
${ }^{14}$ Un rapido esame della quantità di germanismi nella lingua di Dante rispetto a quella moderna, laddove l'Alighieri chiama il castoro bivero -sv. bäver (Inferno XVII, 22)- e l'Austria Osterrichi-sv.Österrike (Inferno, XXXII, 26- ne dà ad esempio immediata caratterizzazione. Per una trattazione dei germanismi nella letteratura italiana delle origini si rimanda ad esempio a Morlicchio (2005).
} 
Anche in Scandinavia, l'ambito geografico in cui principalmente opero, sono in atto progetti che studiano l'intercomprensione a partire da una lingua romanza L2 conosciuta presso discenti che si approcciano a una nuova L3 romanza.

Un quesito a cui ho rivolto la mia attenzione negli anni di didattica universitaria è stato il seguente: è possibile sfruttare almeno in parte un tipo simile di fenomeni anche partendo da una L1 germanica per 1'apprendimento dell'italiano come L2? La risposta che ho trovato empiricamente, e che sarebbe utile in futuro sostanziare ulteriormente con studi di natura quantitativa e qualitativa, è affermativa. Introdotte preliminarmente nozioni di filologia e linguistica storica, è possibile facilitare l'accesso e la ritenzione mnemonica di parte del lessico italiano presso il parlante di lingua madre germanica, e scandinava in particolare.

Nella didattica universitaria dell'italiano L2, impartire nozioni di linguistica storica, anche in ottica extra-romanza, comporta una serie di potenziali vantaggi: oltre alla summenzionata facilitazione in termini di acquisizione di lessico, una simile operazione presso il discente culturalmente germanico è capace di aggiungere sfumature a una visione altrimenti spesso monolitica dell'italiano come linguacultura «latina»; da un punto di vista metodologico, fornisce anche occasione per avviarlo ai rudimenti della linguistica comparata e dell'indoeuropeistica, che sempre più spesso mancano nel percorso di formazione dello studente, soprattutto in Europa settentrionale.

L'attenzione sui germanismi anche nella didattica della lingua italiana all'estero sembra poter gettare quindi un ponte tra la glottodidattica pura e la trasmissione di nozioni di carattere storico-culturale, mettendo in sinergia gli obiettivi pedagogici di "bildning" con quelli di "utbildning",

Come si può realizzare una simile operazione nella pratica? Partiamo dal caso del discente di lingua madre scandinava, generalizzabile poi ad altri discenti di madrelingua germanica con i dovuti aggiustamenti.

Dopo aver introdotto il discente alla tematica dei germanismi nella lingua italiana, e accanto all'invito al ricorso ad un dizionario etimologico per appurare a posteriori la corrispondenza di un germanismo italiano ad un lemma della lingua madre (o dell'inglese, pressoché universalmente conosciuto dalla popolazione studentesca), che è utile per la ritenzione mnemonica del lessico ma non per l'intercomprensione, possiamo favorire il riconoscimento spontaneo di molti germanismi semplicemente fornendo al discente un serie di strumenti, qui già brevemente introdotti nella precedente descrizione dei germanismi della lingua italiana.

In primo luogo si dovranno fornire nozioni di base sui fenomeni storici di trasformazione linguistica interni alle lingue germaniche, in particolare quello della seconda mutazione consonantica, che oltre a rendere individuabili i longobardismi li rende più facilmente riconducibili al corrispettivo scandinavo o inglese che ne è esente ${ }^{16}$.

Si forniranno poi alcuni semplici criteri d'individuazione grafico-fonetica tra quelli precedentemente descritti. Nello specifico la corrispondenza di gu+vocale a un etimo germanico che si presenterà con v/w nella lingua madre o in inglese; l'origine germanica delle

\footnotetext{
${ }^{15}$ Bildung e Ausbildung, per utilizzare i termini tedeschi, più familiari al lettore italiano di quelli svedesi.

16 I fenomeni di trasformazione specifici dell'adattamento del germanismo nella lingua romanza sono ovviamente utili, e possono trovare posto in un corso di linguistica storica, ma non sono strettamente necessari per l'intercomprensione. Lo stesso vale per quelli delle fasi più tarde dello sviluppo delle lingue scandinave.
} 
parole che cominciano con sch+vocale palatale, e quella delle parole che iniziano con zsorda.

Armato di questi strumenti e della coscienza della portata e della presenza dei germanismi nella lingua italiana, il discente di madrelingua scandinava dimostra ${ }^{17}$ capacità di intercomprensione quando affronta un testo contenente lessico per lui precedentemente sconosciuto. Ho voluto più volte in questo scritto indicare, e utilizzare come criterio per la scelta degli esempi, la facile individuazione dei paralleli (con le dovute derive semantiche) tra i germanismi e lingue germaniche moderne, e scandinave in particolare, proprio per rendere quanto più possibile evidente questa possibilità. Mi si permetta ora di ribadirla con un breve testo, ovviamente costruito ad hoc per sondare e allenare il fenomeno, di fronte al quale gli studenti svedesi regolarmente dimostrano capacità di intercomprensione e ritenzione dopo aver ricevuto le nozioni sopra descritte:

Quell'albergo (härbärge) era una stamberga (sten-bärge) blu (blå) nel borgo (borg) in mezzo al bosco (buske), con una sola sala (sal) arredata (in-reda) a mala guisa (vis) ma decorata con ghirlande (girland) e teste di stambecco (stenbock). I guardiani (vårdare) erano una banda (band) di grami (grymma) farabutti (fribytare), una schiera (skara) di manigoldi (man, våld) senza senno (sinne), sempre seduti sulle panche (bänk) vicino al banco (bänk) a trincare (trinken/dricka) e scherzare (scherzen). Il peggiore era Gesualdo (Gesvald/Gisvold), aveva una lunga nappia (näbb) e il ciuffo (tofs) bruno (brun), la barba sempre piena di schiuma (skum) della birra. Presi i guanti (vante) gialli (gul-yellow) dallo scaffale (skåp), e le brache (breeches-brok) bianche (blank) appese alla gruccia (krycka) e uscii in giardino (gården). Camminavo con una stampella (redskap för att stampa fram), avevo male alla schiena (skenben - rak benstruktur som sticker ut i mitten). Era un'onta (hån) da cui non ero riuscito a guarire (värja) dai tempi della guerra (war) quando al guado (ställe där man vadar) non ero riuscito a farmi schermo $(s k a ̈ r m)$.

\section{Conclusioni}

Da questa rapida carrellata si auspica emerga il potenziale ancora da sfruttare dei germanismi della penisola italica, sia come oggetto di studio che come strumento per la didattica universitaria.

Se considerati come oggetti di studio, i lavori fondamentali che storicamente hanno gettato le basi della trattazione filologica sui germanismi sono ad esempio quelli di Galimscheg, Rohlfs, Tagliavini, Bonfante e Pfister, tutti datati tra poco prima della metà e la fine del secolo scorso. Il filone di studi è proseguito specializzandosi ed è tuttora assai attivo. $\mathrm{Ne}$ do esempio menzionando studiosi come Maria Giovanna Arcamone, alla cui produzione scientifica su onomastica e toponomastica germanica in Italia rimando per un approfondimento aggiornato. Una raccolta sistematica dei germanismi è curata tuttora da Elda Morlicchio, che, oltre ad una ampia produzione di studi di onomastica, nell'ambito del progetto del «Lessico Etimologico Italiano» tra il 2000 e il 2016 ha prodotto i nove fascicoli del primo volume di quattro, che in prospettiva si dovranno realizzare.

Come anche questo scritto ha voluto sottolineare, rimangono ancora diverse prospettive aperte sia nello studio che nell'utilizzo dei germanismi penetrati nella penisola italica.

\footnotetext{
${ }^{17}$ Nella pratica didattica di chi scrive e di diversi suoi colleghi.
} 
Nella ricerca, li si può fruttuosamente impiegare come strumenti e oggetti di analisi in vari ambiti, qui rapidamente delineati, e rimangono ancora da mettere in sinergia i crescenti lavori quantitativi con nuovi lavori di tipo qualitativo.

I quesiti che poneva Bonfante nel 1977 "Che parole abbiamo mutuato dai Germani? Per che motivo? Che differenza di cultura notiamo tra i popoli germanici? Cosa apprendiamo sul rapporto tra i Latini e i Germani? Sono problemi complessi su cui non è detta l'ultima parola" (Bonfante 1977, 32). Alcuni hanno ricevuto parziale risposta, ma rimangono tuttora attuali, e ad essi possono aggiungersene altri.

Accanto agli studi linguaculturali emerge una possibilità di proficuo impiego dei germanismi nella lingua italiana anche in ambito glottodidattico ${ }^{18}$ : questa è già stata sondata empiricamente dall'autore, che si augura con il presente scritto di diffonderne forse la pratica ai colleghi, segnalando anche la necessità e l'interesse di studi futuri per quantificarne più sistematicamente l'impatto sia in termini di intercomprensione che di ritenzione mnemonica presso i discenti di madrelingua germanica, e scandinava in particolare.

In questo contesto, riportare l'attenzione sui germanismi anche in veste di strumento didattico, si spera sia inoltre un passo per integrare il recupero, a mio avviso auspicabile, di una impostazione più tradizionalmente filologica nell'insegnamento della linguacultura italiana all'estero, procurando al discente una visione accuratamente e ulteriormente sfaccettata e aprendo prospettive più ampie nella sua comprensione dei sistemi linguaculturali e delle loro evoluzioni nello spazio e nel tempo.

\section{Bibliografia}

Albano Leoni, Federico. 1983. "Bilinguismo e coscienza del bilinguismo nell'Italia Longobarda" in Italia linguistica. Idee, storia, strutture, Bologna; Il Mulino, 133-148

Arcamone, Maria Giovanna. 2006. "Die italienische Anthroponymie germanischen Ursprungs und ihr Fortleben im heutigen Italien", in Name und Gesellschaft im Frühmittelalter. Personennamen als Indikatoren für sprachliche, ethnische, soziale und kulturelleGruppenzugehörigkeiten ihrer Träger, Ed. D. Geuenich e I. Runde, 137-152, Hildesheim-Zürich-New York: Olms

Arcamone, Maria Giovanna. 2006. "Die langobardische Toponomastik zwischen Germania und Romania." in Proceedings of the 21st International Congress of Onomastic Sciences,Uppsala,19-24 August 2002, ed. E. Brylla e M. Wahlberg, 21-33. Uppsala: SOFI

Bonfante, Giuliano. 1977. Latini e Germani in Italia. Bologna: Pàtron.

Cortelazzo, Manlio e Zolli, Paolo. 1999. Il nuovo etimologico DELI. Dizionario Etimologico della Lingua Italiana, II ed. Bologna: Zanichelli

Équipe EuRom5, 2017. http://www.eurom5.com/ Ultimo accesso 10-12-2017

Gamillscheg, Ernst. 1937. Immigrazioni germaniche in Italia. Leipzig: Keller

\footnotetext{
${ }^{18}$ Sicuramente dell'italiano L2 presso discenti di madrelingua germanica o scandinava, ma anche presso discenti di lingua germanica L2 di madrelingua italiana, altro ambito a cui ho recentemente rivolto attenzione e che ribadisce a mio avviso l'universale utilità ed applicabilità di una base di approccio filologico e comparatistico nei corsi offerti dai nostri atenei.
} 
Gamillscheg, Ernst. 1970. Romania Germanica, neu bearbeitete Aufl. Berlin: de Gruyte

Hellquist, Elof. 1989. Svensk etymologisk ordbok, 3e uppl. Lund: Liber

Mestrelli, Carlo Alberto, 1974. "La terminologia longobarda dei manufatti" in La civilità dei Longobardi in Europa, Roma: Accademia Nazionale dei Lincei, pp. 257-269

Molinari, Maria Vittoria. 1987. La filologia germanica. Bologna: Zanichelli

Morlicchio, Elda. 1985. Antroponimia longobarda a Salerno nel IX secolo - I nomi del Codex diplomaticus Cavensis, Napoli: Liguori

Morlicchio, Elda. 2000-2016. "Germanismi, Vol.1, fasc.1-9” in Lessico Etimologico Italiano. Wiesbaden: Ludwig Reichert Verlag

Morlicchio, Elda. 2001. "Migrazioni di popoli e di parole: l'eredità linguistica dei Germani in Italia", in Società multiculturali nei secoli V-IX. Scontri, convivenza, integrazione nel Mediterraneo occidentale, a c. di M. Rotili, Atti delle VII giornate di studio sull'età romanobarbarica (Benevento), Napoli, pp.109-125.

Morlicchio, Elda. 2005. "Riflessioni per lo studio del plurilinguismo nel contesto medievale", in Il plurilinguismo in area germanica nel Medioevo, Atti XXX Convegno Associazione Italiana di Filologia Germanica (Bari 2003), c. di L. Sinisi, Bari, pp. 211-225

Morlicchio, Elda. 2005. "Germanismi nella letteratura italiana delle origini", in Lessico, parole-chiave, strutture letterarie del Medioevo romanzo, a c. di S. Bianchini, Roma: Bagatto Libri, pp. 319-373.

Morlicchio, Elda, 2011. "Dinamiche sociolinguistiche nell'Italia delle invasioni barbariche", in C. Ebanista e M. Rotili (a c. di), Archeologia e storia delle migrazioni. Europa, Italia, Mediterraneo fra tarda età romana e alto medioevo. Convegno internazionale di studi, Cimitile / Santa Maria Capua Vetere, 17-18/06/2010, Cimitile, Tavolario Edizioni, pp. 217-228

Morlicchio, Elda. 2012. "I documenti notarili alto-medievali campani e gli studi di linguistica e onomastica germanica", in R. Sornicola e P. Greco (a c. di), La lingua dei documenti notarili alto-medievali dell'Italia meridionale. Bilancio degli studi e prospettive di ricerca, Napoli:Tavolario edizioni, pp. 177-190

Pepe, Gabriele.1942. Il Medioevo barbarico d'Italia, II ed. aumentata. Torino: Einaudi

Pfister, Max. 1998. Trasformazioni lessicali e società nella crisi tra tardo antico e alto medioevo in Morfologie sociali e culturali in Europa fra tarda antichità e alto medioevo (3-9 aprile 1997) Settimane di studio del centro italiano di studi sull'alto medioevo 45 (1998), 1107-1150 (Diskussion: 1151-1155). Spoleto: Centro Italiano di Studi sull'Alto Medioevo

Rohlfs, Gerhard. 1947. Germanisches Spracherbe in der Romania. München

Tagliavini, Carlo. 1969. Le origini delle lingue neolatine. Bologna: Pàtron. 